\title{
Developing Slow Sensor for Slow Design
}

\author{
Teng-Wen Chang, ${ }^{1 *}$ Yi-Sin Wu, ${ }^{2}$ Sambit Datta, ${ }^{3}$ and Wei-Lung $\mathrm{Mao}^{4}$ \\ ${ }^{1}$ Dept. of Digital Media Design, National Yunlin University of Science and Technology, \\ No. 123, Sec. 3, University Rd., Douliu, Yunlin 640, Taiwan (R.O.C.) \\ ${ }^{2}$ Graduate School of Design, National Yunlin University of Science and Technology, \\ No. 123, Sec. 3, University Rd., Douliu, Yunlin 640, Taiwan (R.O.C.) \\ ${ }^{3}$ Curtin Institute of Computation, Curtin University, Kent St., Bentley, Western Australia, 6102, Australia \\ ${ }^{4}$ Dept. of Electrical Engineering, National Yunlin University of Science and Technology, \\ No. 123, Sec. 3, University Rd., Douliu, Yunlin 640, Taiwan (R.O.C.)
}

(Received November 18, 2019; accepted June 5, 2020)

Keywords: gardening activity, slow sensor, ambient interaction, slow technology

Slow design using slow technology in the domain of human-computer interaction is often utilized to reflect people's attachment to behaviors or objects and to promote positive changes in people's settling behaviors and the meaning behind them. In this paper, we propose a three-step slow sensor model for developing a slow sensor for classifying the behaviors of the elderly during gardening activities. Step 1: Finding daily objects and a suitable slow activity using the behavior setting. Step 2: System modeling with the corresponding sensors set to collect data. Step 3: Data modeling by designing data schema and behavioral classification. An experiment using a prototype slow sensor, gardening as the slow activity, and IoT with sensor network technology is conducted and data are collected and analyzed to determine the daily incremental activities of the elderly. The results are evaluated and validated for behavioral classification after two weeks of experimentation.

\section{Introduction}

Slow design ${ }^{(1)}$ using slow technology in the domain of human-computer interaction is often utilized to reflect people's attachment to behaviors or objects and to promote positive changes in people's settling behaviors and the meaning behind them. Additionally, slow design is also used to study people's participation, emotion, and intention in the interaction. ${ }^{(2)}$ Therefore, a slow sensor for collecting slow activity information is a crucial part of slow design.

Although slow design is currently not well defined, most cases share a common pattern in terms of sensor design, that is, (1) human behavior in slow design is usually represented by data accumulated over a period of time using a group of behavior-related sensors, and (2) these types of data to be collected are accumulated, complex, and often multimodal, representing condensed information of situations requiring response data analysis to elucidate behavior features. In this paper, a slow sensor model within the setting of gardening activity of the elderly, based on the "behavior setting" concept, ${ }^{(3,4)}$ is proposed.

\footnotetext{
*Corresponding author: e-mail: tengwen@gemail.yuntech.edu.tw
} https://doi.org/10.18494/SAM.2020.2811 
In the proposed slow sensor model, the following three sequential steps are given towards the realization of the slow design objective, which, in this work, is the gardening activity of the elderly (Fig. 1).

1. Find daily objects with suitable activity: Observe the relationship between the user's activity over a long period of time and explore the slow activity that will provide the desired interaction with the surrounding life features and objects in line with a given slow design objective.

2. Set sensors to collect data: Install the appropriate sensors in objects associated directly with the activities identified above; appropriate sensors are selected and data are determined in accordance with the continuity or phase of the activity.

3. Design the data schema and behavioral classification: Refine the activity and sensors through data schema design for the accumulated data. Additionally, the behavioral classification in accordance with the given slow design objective is applied in the schema design. If the behavioral classification is insignificant, return to step 2 and replace the sensor. If the behavioral classification is successful but does not fulfill the slow design objective, return to step 1 and determine other activities or daily objects that can provide sufficient accumulated data.

\section{Related Approach}

We reviewed the literature on slow technology and smart homes to derive the meaning of and guidelines for designing slow sensors. Slow technology is often applied to the elderly to promote their well-being, which is associated with slow activity in our study. ${ }^{(5,6)}$ Smart home technology while based on Internet of Things (IoT) brings interoperability and interconnection to daily objects in the surroundings of elderly. ${ }^{(7-9)}$ It provides assistance to the elderly; this is the basis of the connecting functionality in the slow sensor model. Providing support for elderly people who live alone with a high degree of independence is the setting for the slow sensor developed in this study. ${ }^{(10)}$

Furniture and other items, such as chairs, beds, gates, and watering tools, equipped with IoT and a sensor network have a high association with the life of the elderly. ${ }^{(11)}$ Sensors set on such daily objects transmit signals in accordance with the time of activity of the elderly. The possible
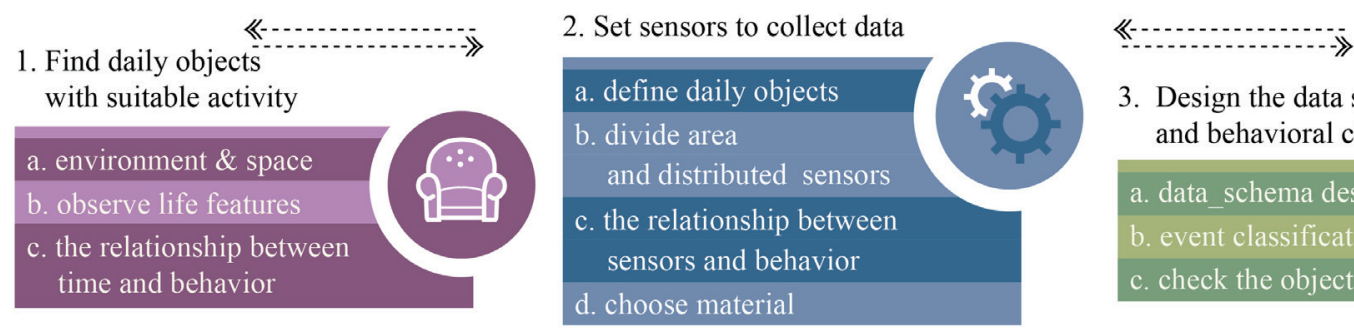

3. Design the data schema and behavioral classification

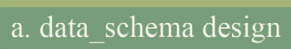

b. event classification c. check the objective

Fig. 1. (Color online) Process of realizing slow design objective. 
sensors are ultrasonic, sound, flexible, and radio frequency identification (RFID) sensors. In addition to the daily objects, social behaviors are also often recorded; the social activity of each family member can be classified as slow interaction. ${ }^{(12)}$

Data were accumulated through two experiments of slow design: developing an interaction game between elderly subjects and their family members incorporating gardening activities and pairing elderly subjects with volunteers in daily life activities, such as buying groceries or replacing a light bulb..$^{(13,14)}$ The system flow of slow sensor design was established and analyzed to refine the model presented in this paper.

An experiment is conducted to verify the proposed slow sensor model, and the system and data modeling based on the slow sensor model are described below.

\section{System Modeling}

The slow design objective of the experiment is to provide a classification of routine behavior to increase the amount of positive interaction for the elderly over a long period of time. According to a survey on possible activities, gardening is one of the popular leisure activities for the elderly. ${ }^{(15)}$ Therefore, in step 1 of the slow sensor model, gardening is selected as the suitable activity with a plant pot, i.e., the pot as shown in Fig. 2, as the daily object with IoT connectivity.

In the second step, the pot is equipped with a breadboard with Arduino UNO (parts 1 and 2), two pressure sensors (part 3), a temperature and humidity sensor (part 4), and an ultrasonic sensor (part 5). The sensors are placed throughout the pot at the positions indicated by the corresponding number in the figure to record the gardening activities. The accumulated data are uploaded to a cloud database. The MS5803-14BA pressure sensors used in this gardening

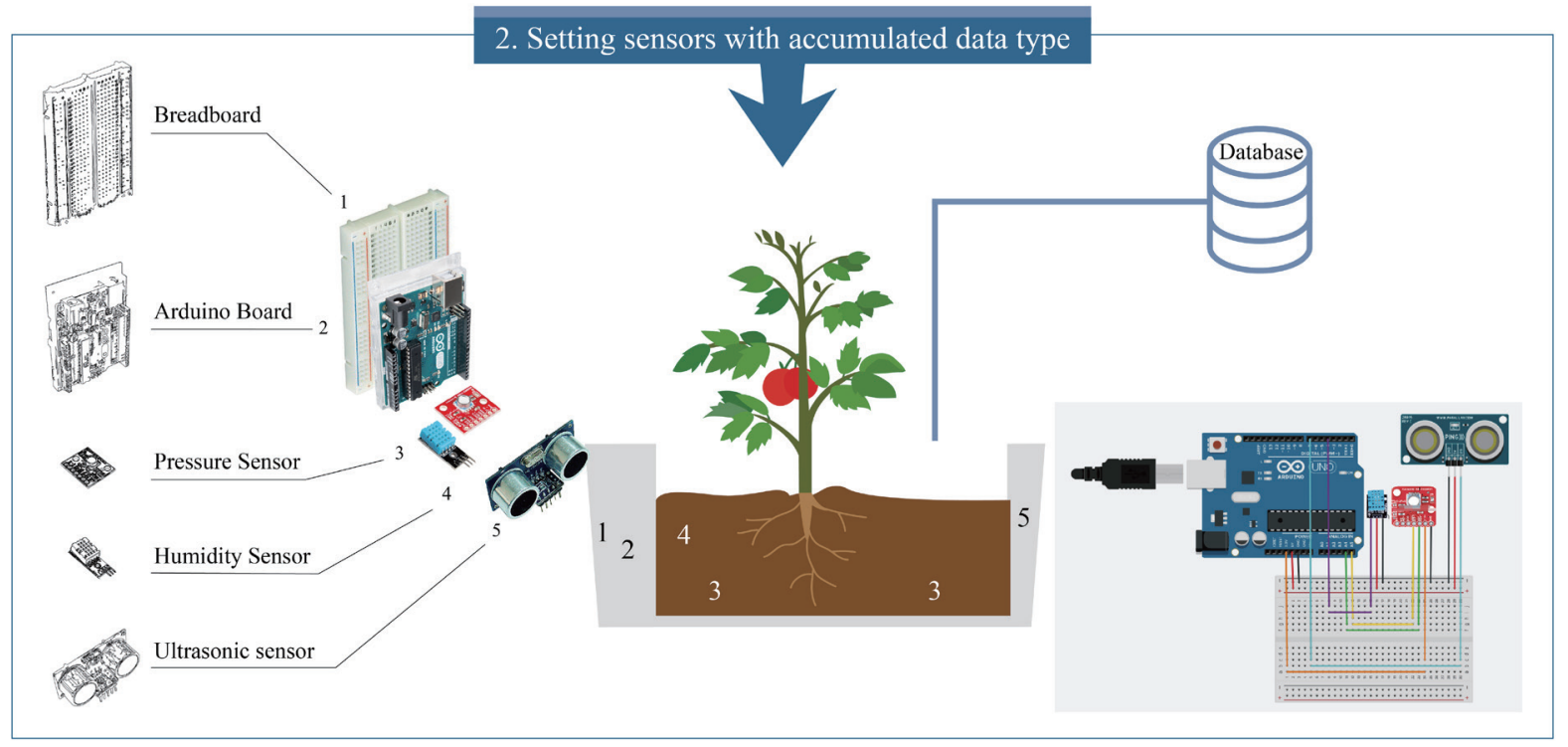

Fig. 2. (Color online) Gardening activity module in step 2. 
activity module are characterized by low power consumption and long-term stability. They are fully sealed, making them suitable for outdoor installation, and the use of waterproof glue and an antimagnetic stainless-steel ring allows the sensor to withstand a water pressure of 30 bar. The BME680 IAQ sensor module for temperature and humidity is a small device with a temperature measurement range between -40 and $85{ }^{\circ} \mathrm{C}$ and a humidity measurement range between 0 and $100 \%$. The data obtained by the sensor are transmitted to the Arduino where the average is calculated and then uploaded to the database.

\section{Data Modeling}

Next, data modeling (step 3) is conducted. The data of two weeks of gardening activity are accumulated from the gardening activity module described above. The data upload architecture is based on the multipath routing of a software-defined network (SDN) combined with segment routing (SR) (right side of Fig. 3). ${ }^{(16)}$ In this database schema design for the analysis of the slow behavior information and their application, the accumulated data are divided into two types: EnvironmentSensor and Event.

Behavior classification is computed using the accumulated data. In gardening activity, the user's information (elderly) is imported into the database. The recorded events in the gardening activity are defined in step 1 as watering, stay, and raining. When multiple sensors sensed the data, that data can be classified as watering, stay, or raining using the logic defined as the pseudocode in Table 1.

This pseudocode is for classifying watering and describes the process of analyzing the required information and the rationale for the use of classifiers for each recorded event. First, the data value from the sensor is declared, and the difference between the previous and current values is calculated. Then, it is determined whether the difference is greater or less than the critical value used as the basis for classification. The critical value for humidity is 30 . When the distance value is greater than 90 and the humidity is greater than 30 , the behavior is "watering".
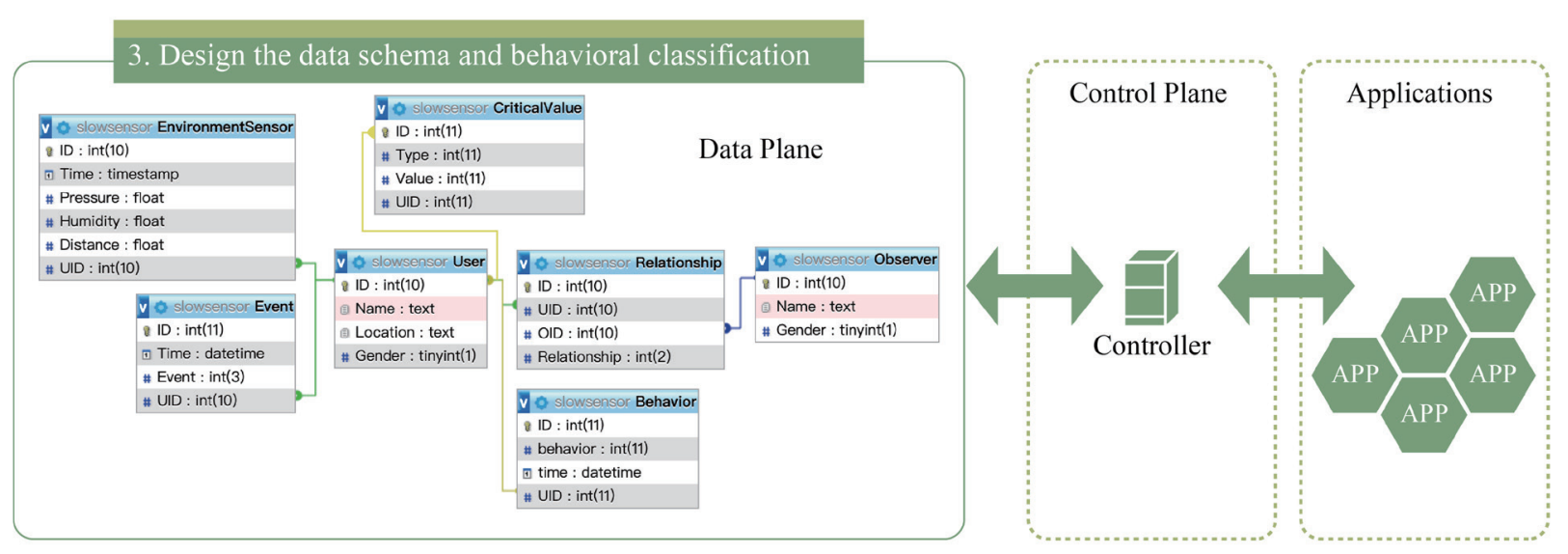

Fig. 3. (Color online) Database schema for the storage of slow-sensor data used in analysis of human behavior. 
Table 1

Pseudocode for classifying watering.

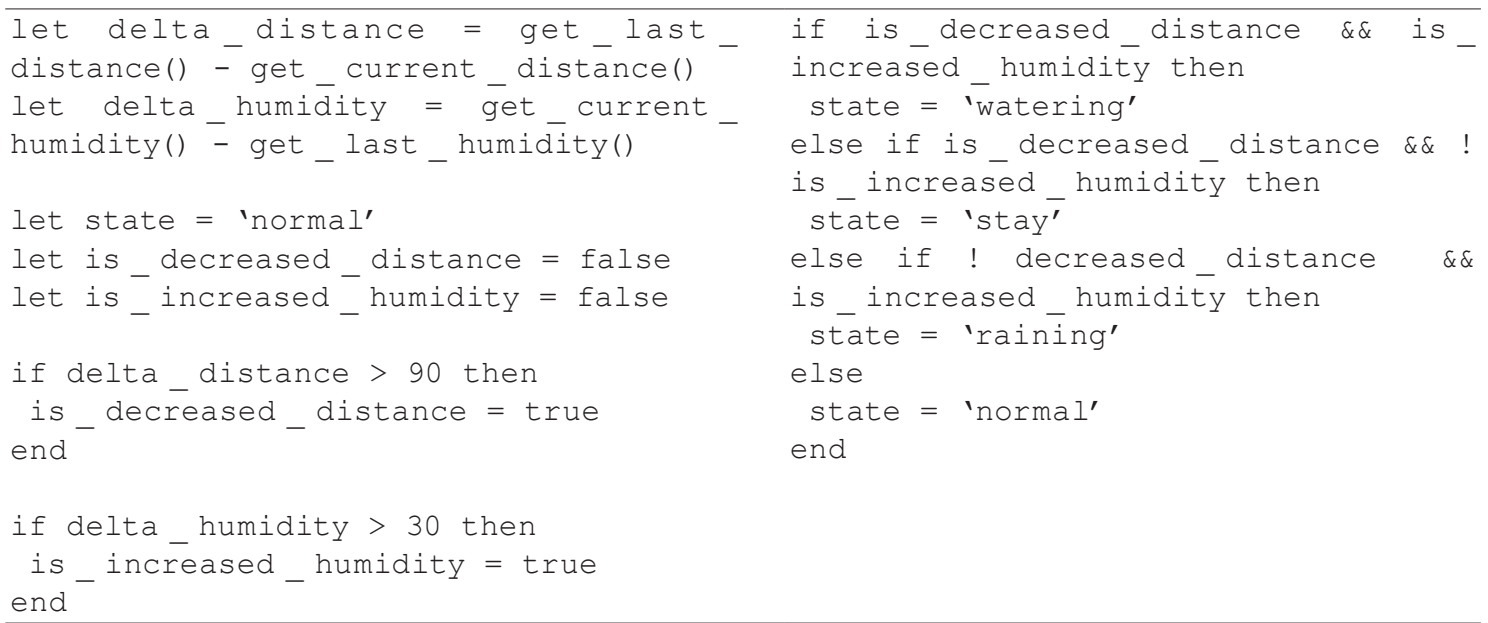

When the distance value is no greater than 90 and the humidity is no greater than 30 , the behavior is "stay in place".

\section{Results and Discussion}

In this experiment, we focused on gardening activities of an elderly subject and developed a slow sensor based on the model proposed previously. On the basis of the linear behaviors classified, the normal and abnormal changes can be identified and noted. The slow sensor is a suitable tool for data collection, but the data collected using a common sensor are meaningless for the direct and absolute interpretation of behavior. Therefore, a corresponding data schema for analyzing the meaning behind the data is needed.

Because the slow sensor is set for a specific behavior, there will be much idle time, so the sensor is adjusted to upload data every second, and the data of $30 \mathrm{~min}$ are averaged. In this way, the time period or time interval can be better determined. Figure 4 shows the outcomes of daily analyses of gardening activity data.

A meaningful time frame has been selected, and the occurrence of an actual behavior is indicated by the highlighted area. Humidity and pressure values are the judgement factors of event model 1, and the distance, given by the ultrasound value, is the judgement factor of event model 2. For the analysis, only significant factors are retained (i.e., humidity value $>30$ ). The daily analyses reveal that the activity of the subject increases at 06:00, and also at 17:00, when the subject enters the measurement area again. Data were collected from the three sensors for two weeks, and from these data, we can speculate on the lifestyle and condition of the subject by referring to the results of the preliminary analysis. 


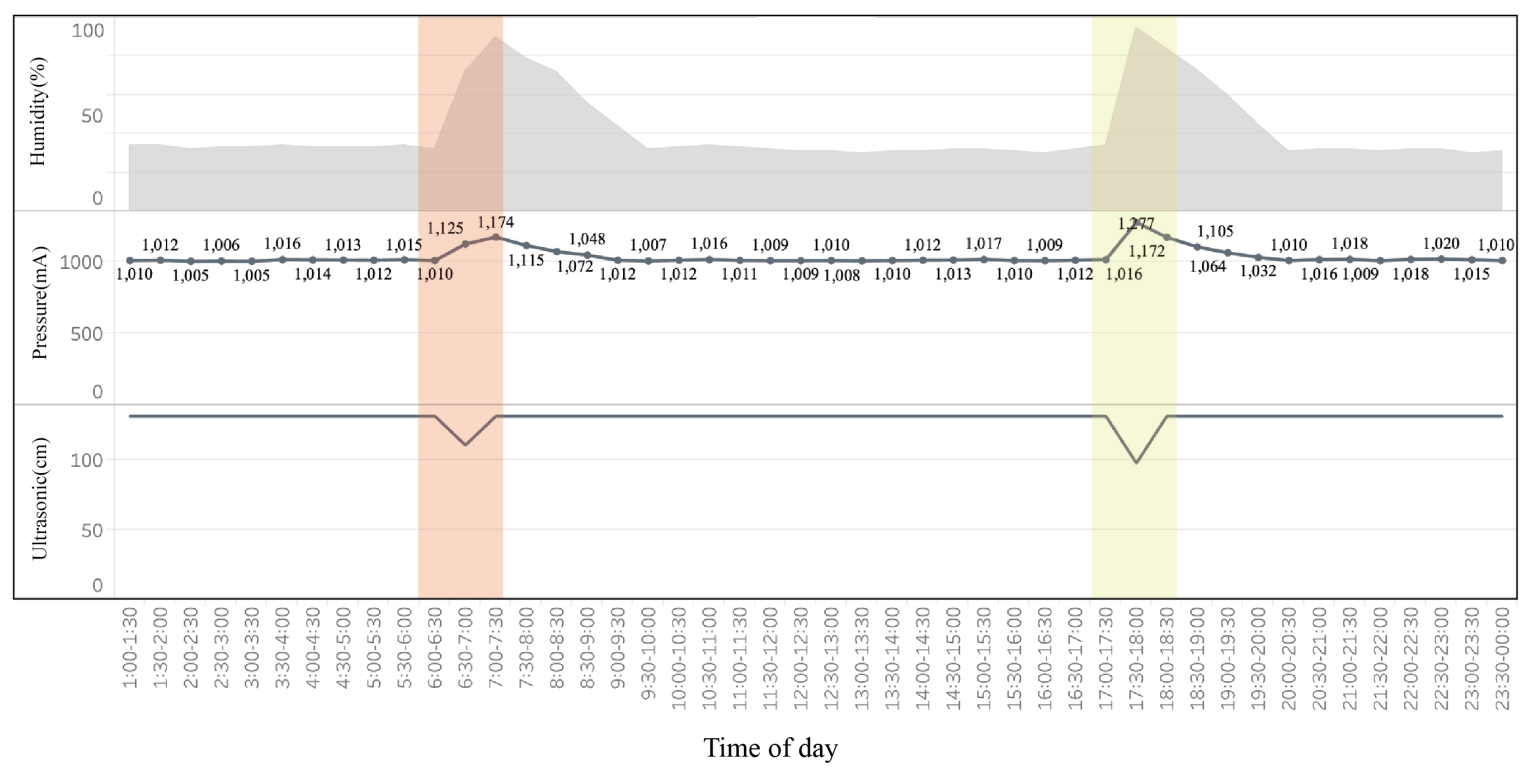

Fig. 4. (Color online) The accumulated data reflects the gardening activity of the subject.

\section{Conclusions}

We proposed a "slow sensor model design process" with an accumulated database and a representation of the resultant information formats. The first prototype of the slow sensor model was developed for the elderly performing gardening activities. The multimodal sensor system includes a temperature sensor, a humidity sensor, two pressure sensors, and one ultrasonic sensor in a daily object, i.e., a plant pot. EnvironmentSensor and Event data were collected following the designed data schema and their classifiers for behavior classification. In conjunction with the IoT and sensor network from smart home technology, the slow sensor model for accumulating information over a long period of time can help reveal the life patterns and conditions of the elderly. It can also be quickly adjusted within the constraints of the environment and the slow design objectives.

By visualizing the information of gardening activity, we can see the exact relationship between behavior and daily objects. However, more complex environments and human behaviors should be considered. In future work, in addition to adjusting the model to accommodate a number of people and a range of environments, the system will be deployed and tested in the homes of five subjects to technically validate and assess its impact on human behavior.

\section{Acknowledgments}

This study was partly supported by the Ministry of Education of the Republic of China (Taiwan) under the Teaching Innovation Project on an Issue-Oriented Approach to Narrative Competence Development. 


\section{References}

1 B. Grosse-Hering, J. Mason, D. Aliakseyeu, C. Bakker, and P. Desmet: Proc. SIGCHI Conf. Human Factors in Computing Systems (2013) 3431.

2 D. Fallman: Proc. SIGCHI Conf. Human Factors in Computing Systems (2011) 1051.

3 R. G. Barker and H. F. Wright: Child Dev. 20 (1949) 131. https//doi.org/10.2307/1125869

4 K. Lewin: Field Theory in Social Science, D. Cartwright, Ed. (New York: Harper, 1951).

5 National Institute on Aging (NIA): World's older population grows dramatically: https://www.nih.gov/newsevents/news-releases/worlds-older-population-grows-dramatically (accessed May 2020).

6 H. Wan, D. Goodkind, and P. Kowal: Int. Popul. Rep. 1 (2016) 95.

7 B.-R. Chang, H.-F. Tsai, J.-L. Lyu, and T.-K. Yin: Sens. Mater. 31 (2019) 3485. http://doi.org/10.18494/ SAM.2019.2563

8 D. P. Rubanga, K. Hatanaka, and S. Shimada: Sens. Mater. 31 (2019) 831. https://doi.org/10.18494/ SAM.2019.2154

9 Y.-H. Chang, R.-H. Huang, H.-W. Lin, and P.-L. Chung: Smart home monitoring with context interaction based on a cyber-physical-social system, A. D. K.-T. Lam, S. D. Prior, S.-T. Shen, S.-J. Young, and L.-W. Ji, Eds. (CRC Press, London, 2019) Chap. 5. https://doi.org/10.1201/9780429019777

10 M. Domb: Smart Home Systems Based on Internet of Things: In Internet of Things (IoT) for Automated and Smart Applications, Y. Ismail, Ed. (2019).

11 M.-N. Liao and T.-W. Chang: 6th Int. Conf. Distributed, Ambient, and Pervasive Interactions (DAPI) (Springer, 2015) 317-324.

12 G.-J. Yu and T.-W. Chang: Proc. 14th Int. Conf. Human-Computer Interaction. Towards Mobile and Intelligent Interaction Environments (Springer, 2011) 250-258.

13 Y.-S. Wu, T.-W. Chang, and S. Datta: Archit. Comput. 14 (2016) 263. http://doi.org/10.1177/1478077116663349

14 Y.-S. Wu, T.-W. Chang, Y.-R. He, Y. Wang, W.-H. Chen, and Y.-C. Zhang: Proc. 6th Int. Conf. Distributed, Ambient, and Pervasive Interactions (DAPI) (Springer 2015) 614-624.

15 N. Garatachea, O. Molinero, R. Martínez-García, R. Jiménez-Jiménez, J. González-Gallego, and S. Márquez: Arch. Gerontology Geriatrics 48 (2009) 306. http://doi.org/10.1016/j.archger.2008.02.010

16 C.-B. Lin, Y.-C. Chang, and Y.-C. Chang: Software-defined network for multipath routing of IoT data, A. D. K.-T. Lam, S. D. Prior, S.-T. Shen, S.-J. Young, and L.-W. Ji, Eds. (CRC Press, London, 2019) Chap. 3. https:// doi.org/10.1201/9780429058127

\section{About the Authors}

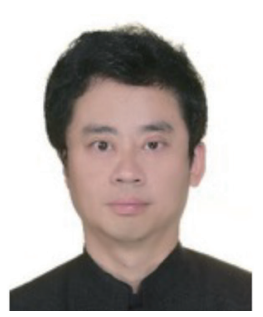

Teng-Wen Chang received his Ph.D. degree from the University of Adelaide in 1999, his M.Arch. degree from the University of Pennsylvania in 1993, and his M.S. degree in computational design from Carnegie Mellon University in 1995. He is currently a professor in the Department of Digital Media Design and Director of SOFTLab at National Yunlin University of Science and Technology, Taiwan. His current research interests are design space exploration, human machine interaction, and sensible intelligent machines. (tengwen@yuntech.edu.tw)

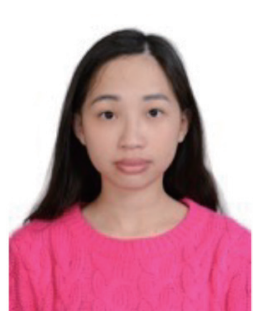

Yi-Sin Wu is a Ph.D. student at National Yunlin University of Science and Technology, Taiwan. She is a human-computer interaction and user experience designer. In 2018, she became a project assistant in crossdisciplinary team building based at Idea Factory (IDF). She and the team members have created interactive devices in Thailand, Beijing, and Suzhou. Since 2015, she has been involved in planning international workshops. (wu.rilla918@gmail.com) 


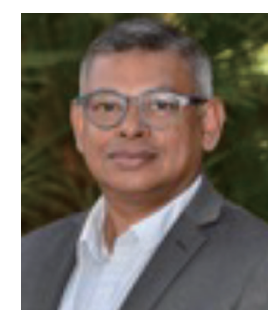

Sambit Datta is Dean International of the Faculty of Science and Engineering at Curtin University. Professor Datta studied architecture at the School of Architecture, Centre for Environmental Planning and Technology (CEPT) in Ahmedabad, India. He completed his Master of Architecture degree at the National University of Singapore and his doctorate in the field of computational design at the University of Adelaide. He was a Michael Ventris Memorial Scholar at the Architectural Association, London.

(sambit.datta@curtin.edu.au)

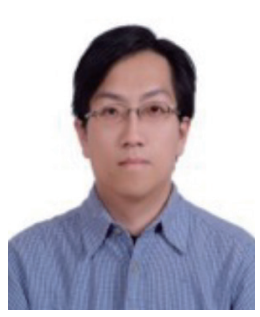

Wei-Lung Mao received his B.S. degree in electrical engineering from National Taiwan University of Science and Technology in 1994 and his M.S. and Ph.D. degrees in electrical engineering from National Taiwan University in 1996 and 2004, respectively. He is a professor in the Department of Electrical Engineering and Graduate School of Engineering Science and Technology, National Yunlin University of Science and Technology. His research interests are precision motion control, intelligent and adaptive control systems, satellite navigation systems, adaptive signal processing, neural networks, and communication electronics. (wlmao@yuntech.edu.tw) 